\title{
BMJ Open Decision-making on the location of care of the elderly: protocol for a systematic review of qualitative studies
}

\author{
Gema Serrano-Gemes, ${ }^{1}$ Rafael Serrano-del-Rosal, ${ }^{2}$ Manuel Rich-Ruiz ${ }^{3}$
}

To cite: Serrano-Gemes G, Serrano-del-Rosal R, RichRuiz M. Decision-making on the location of care of the elderly: protocol for a systematic review of qualitative studies. BMJ Open 2018;8:e022411. doi:10.1136/ bmjopen-2018-022411

- Prepublication history and additional material for this paper are available online. To view these files, please visit the journal online (http://dx.doi. org/10.1136/bmjopen-2017022411).

Received 16 February 2018 Revised 23 May 2018

Accepted 6 September 2018

Check for updates

(C) Author(s) (or their employer(s)) 2018. Re-use permitted under CC BY-NC. No commercial re-use. See rights and permissions. Published by BMJ.

${ }^{1}$ Universidad de Córdoba (UCO), Instituto Maimónides de Investigación Biomédica de Córdoba (IMIBIC), Hospital Universitario Reina Sofía (HURS), Cordoba, Andalucía, Spain ${ }^{2}$ Instituto de Estudios Sociales Avanzados (IESA-CSIC), Consejo Superior de Investigaciones Científicas, Cordoba, Andalucía, Spain

${ }^{3}$ Universidad de Córdoba (UCO), Instituto Maimónides de Investigación Biomédica de Córdoba (IMIBIC), Hospital Universitario Reina Sofía (HURS), CIBERFES (CIBER de Fragilidad y Envejecimiento Saludable), Cordoba, Spain

\section{Correspondence to} Gema Serrano-Gemes; gemaserranogemes@hotmail. com

\section{ABSTRACT}

Introduction The elderly must take part in the management of their own health. One of the aspects they should be able to decide on is the place where they want to live. The aim of this review is to synthetise qualitative evidence in order to understand how decisions are made on the location of care of the elderly.

Methods and analysis Systematic review of qualitative studies. Six databases have been consulted: Web of Science, PubMed, Scopus, CINAHL Complete, PsycINF0 and SciELO Citation Index (from the beginning to 29 November 2017). The inclusion criteria will be: studies that deal with the decision-making process on the location of care of the elderly (already experienced by the participants), original studies, qualitative or mixed-method studies and studies written in English or Spanish. The obtained results will be exported to the Zotero bibliography manager. The references will be reviewed by title and abstract and, later, the complete texts will be reviewed for their inclusion. A tool created for this study will be used to extract the data. The quality will be assessed with Critical Appraisal Skills Programme Español. The data synthesis will be carried out using the constant comparative method. All this process will be performed independently by two reviewers. Enhancing transparency in reporting the synthesis of qualitative research has been used to draw up this protocol.

Ethics and dissemination This protocol did not require ethical approval, since it is a protocol for a systematic review. The plans to disseminate our results include publishing a research paper in a high-impact journal in our study area. Also, if possible, our results will be presented in scientific conferences. Besides, the obtained results will complement and discuss the doctoral thesis of one of the authors of the review.

PROSPERO registration number CRD42018084826.

\section{INTRODUCTION}

Ageing has turned into an essential political question, due to the fact that both the proportion and the number of elderly people are increasing significantly worldwide. ${ }^{1}$ The ageing of populations all around the world will considerably increase the quantity of elderly people who are care-dependent. ${ }^{1}$

However, it is striking that the current healthcare systems and services do not

\section{Strengths and limitations of this study}

The results of this study will widen the knowledge and inform future actions on the decision-making process in the field of location of care of the elderly.

- The findings achieved in this study will help both researchers and those people involved in this decision-making process.

- This study will help to improve the field of study of systematic reviews of qualitative studies, as the final report will inform about all the steps taken to carry out this systematic review.

properly suit the elderly's individual preferences and diverse health needs ${ }^{12}$ which is the case across the world. ${ }^{1}$

In order to respond to the specific needs of this population, it will be necessary to adapt the healthcare services, placing the elderly in the centre of healthcare, ${ }^{1-3}$ thus being necessary to include them as active participants in the planning of healthcare ${ }^{13}$ and in managing their own health. ${ }^{1}$

To this lack of adaptation of healthcare systems and services, it is necessary to add the scarce existing literature about decision-making and ageing. ${ }^{4}$ This literature usually focuses on the different aspects that somehow hinder the participation of the elderly in their own healthcare.

These aspects are usually problems linked to the ageing process: cognitive $^{56}$ and physical impairment. ${ }^{6} 7$ The elderly's unwillingness to participate is also mentioned, ${ }^{568}$ with this responsibility thus falling on the family ${ }^{58}$ or on the doctor. ${ }^{6-8}$ Finally, another aspect presented as problematic for the elderly is the discrimination on the grounds of age in healthcare services. ${ }^{1}$

One of the aspects where the elderly should have control and the right to decide is the place where they want to live. ${ }^{13}$ Moreover, the ability of making their own decisions regarding the place to live has been considered by the elderly in a recent study as being 
very important for them, ${ }^{9}$ although, at the same time, this decision is also considered as involving high emotional stress. $^{4}$

All of this justifies and leads to our main objective: to synthesise the existing evidence with qualitative methodology in order to achieve a deep understanding of how decisions are made on the location of care of the elderly. In order to reach this objective, this review has based its research question and subsequent search strategy on a structure of key elements that is specific for qualitative reviews: Population, Phenomena of Interest and Context ${ }^{10}$ - the elderly, decision-making process on the location of care, and decisions not linked to death, intellectual disabilities, substance abuse, acute care or temporary locations of care, respectively.

Therefore, our research question would be: how is the location of care of the elderly decided on? In turn, this question will be specified into the following aspects: (1) Who takes part in the decision about the location of care of the elderly? (2) How do the participants experience the decision-making process on the location of care? (3) What are the participants' motives/reasons to decide on the location of care?

After searching in the Cochrane Library, Joanna Briggs Institute Systematic Review Databases and the International prospective register of systematic reviews (PROSPERO), no systematic reviews or systematic review protocols were found tackling this matter; therefore, it was decided to carry out a systematic review on the decision-making process on the location of care of the elderly. This protocol has been registered in PROSPERO.

\section{METHODS \\ Design}

Systematic review of qualitative studies.

It was decided to only study qualitative studies, due to the nature of the main objective of this research: to achieve a deep understanding of how the decisions on the location of care of the elderly are taken, since qualitative research is the type of research which may more efficiently and appropriately provide the necessary information to be able to answer our research question.

It is important to point out that in order to draw up this protocol, the work by Butler $e t a l,{ }^{11}$ a guide to draw up protocols of systematic qualitative reviews, and the Enhancing transparency in reporting the synthesis of qualitative research (ENTREQ) statement ${ }^{12}$ have been used as informative support. At the same time, the Preferred Reporting Items for Systematic Reviews and Meta-Analyses Protocols (PRISMA-P) statement ${ }^{13}$ (see online supplementary additional file 1) has also been used and completed to prepare this systematic review protocol.

\section{Information sources}

The consulted databases have been Web of Science (core collection of Web of Science), MEDLINE (through PubMed), Scopus, CINAHL Complete (through
EBSCOhost), PsycINFO (through ProQUEST) and SciELO Citation Index (through Web of Science) (from the beginning to 29 November 2017).

In addition, the references in the papers which will be finally included will also be reviewed.

We expect to finish the review by autumn of 2018 .

\section{Search strategy}

The search strategy has focused on five key concepts which are made up of different terms. The terms used to refer to each concept were linked using the connective 'OR', and then the four main concepts were linked using the connective 'AND'; lastly, the connective 'NOT' was used to link these four concepts to the fifth one. The subject headings were used when necessary, and the terms were adjusted to the different databases used. The different search strategies used in each database are shown in table 1 .

\section{Eligibility criteria}

In order to contextualise the framework where this systematic review of qualitative studies will be carried out, it is essential to describe how some concepts of interest are going to be dealt with and understood throughout this review:

\section{The elderly}

In this research, the concept of elderly person will include all the people who are 65 or older, both having cognitive/ physical impairment or problems or not. This decision was taken because in the literature review done by Smith and Crome ${ }^{14}$ about the relocation, it is said that not all studies dealing with it exclude people with more physical and cognitive impairment. In fact, the results of Dickinson's study ${ }^{15}$ pointed out that, except for the elderly suffering from more severe impairment, the elderly with different degrees of memory impairment were able to preserve knowledge about their relocation.

\section{Decision-making process}

People tackle the decision-making process from a historical, social, interpersonal and cultural context. ${ }^{16}$ That is why, for this review, it has been decided to focus on three aspects that the authors consider crucial for this process: who takes part in the decision, how they go through this decision-making process and the motives and/or reasons to make the decision.

Due to this, no type of specific informer has been specified, in order to be able to respond in a wider manner to the aim of our review, focusing on discovering who the people who take part in the decision are. In this way, it will be possible to analyse not only what type of people/ group takes part, but also to study if there is more than one participant, as well as the interrelations which are created throughout this decision-making process.

In addition, this systematic review focuses on the complete decision-making process, not only on the final decision. This is why, throughout the study, the different options chosen for the location of care will not be 
Table 1 Databases and search terms used to identify literature for review

\begin{tabular}{|c|c|c|}
\hline Database & Search terms & $\begin{array}{l}\text { No of } \\
\text { articles }\end{array}$ \\
\hline AND & $\begin{array}{l}\text { ("Decision Making"[Mesh:NoExp] OR Consensus [MeSH Terms] OR Uncertainty [MeSH Terms] OR "Choice } \\
\text { Behavior"[Mesh:NoExp] OR Dissent and Disputes [MeSH Terms] OR "Negotiating"[Mesh:NoExp] OR Patient } \\
\text { Participation [MeSH Terms] OR "Decision Making" OR "Patient Participation" OR "Patient Involvement" OR } \\
\text { "Patient Engagement") }\end{array}$ & \\
\hline AND & (“Placement" OR “Location of care" OR “Relocation” OR "Relocating" OR “Transition”) & \\
\hline NOT & $\begin{array}{l}\text { (Intellectual Disability [MeSH] OR Substance-Related Disorders [MeSH] OR "Mental Retardation" OR "Mental } \\
\text { Deficiency" OR "Drug Dependence" OR "Drug Addiction" OR "Substance Abuse" OR "Drug Abuse" OR } \\
\text { Palliative care [MeSH] OR Terminal care [MeSH] OR Life support care [MeSH] OR Advance care planning } \\
\text { [MeSH] OR "Palliative care" OR "Terminal care" OR "End of life care" OR "Hospice care" OR "Life support } \\
\text { care" OR "Advance care planning" OR "Advance directives" OR "Place of death") }\end{array}$ & \\
\hline AND & $\begin{array}{l}\text { ((MH "Decision Making") OR (MH "Consensus") OR (MH "Decision Making, Clinical") OR (MH "Decision } \\
\text { Making, Ethical") OR (MH "Decision Making, Family") OR (MH "Decision Making, Patient") OR (MH "Dissent } \\
\text { and Disputes+") OR (MH "Consumer Participation") OR "Patient participation" OR "Patient involvement" OR } \\
\text { "Patient engagement" OR "Decision Making") }\end{array}$ & \\
\hline AND & ((MH "Relocation") OR "Relocating" OR "Location of care" OR "Placement" OR "Transition" OR "Relocation") & \\
\hline AND & $\begin{array}{l}\text { ((MH “Community Living") OR (MH “Assisted Living") OR (MH "Institutionalization") OR (MH “ Housing for the } \\
\text { Elderly") OR (MH "Residential Facilities") OR "Independent living" OR "Aging in Place" OR "Institutionalization") }\end{array}$ & \\
\hline NOT & $\begin{array}{l}\text { ((MH "Intellectual Disability+") OR (MH "Substance Use Disorders+") OR "Mental Retardation" OR "Mental } \\
\text { Deficiency" OR "Drug Dependence" OR "Drug Addiction" OR "Drug Abuse" OR "Substance Abuse" OR } \\
\text { (MH "Terminal Care+") OR (MH "Life Support Care+") OR (MH "Advance Care Planning") OR (MH "Advance } \\
\text { Directives+") OR "Terminal Care" OR "Palliative Care" OR "Hospice Care" OR "Life support care" OR "Advance } \\
\text { care planning" OR "Advance directives" OR "End of life care" OR "Place of death") }\end{array}$ & \\
\hline AND & (“Relocation" OR “Relocating" OR “Location of care" OR "Placement” OR “Transition”) & \\
\hline AND & $\begin{array}{l}\text { (SU.EXACT(“Assisted Living") OR SU.EXACT(“Retirement Communities") OR SU.EXACT(“Aging in Place") } \\
\text { OR SU.EXACT(“Institutionalization”) OR SU.EXACT("Residential Care Institutions") OR SU.EXACT("Nursing } \\
\text { Homes") OR "Independent living" OR "Aging in place" OR "Institutionalization”) }\end{array}$ & \\
\hline NOT & $\begin{array}{l}\text { ("Palliative Care" OR "Terminal care" OR "Hospice care" OR "Life support care" OR "End of life care" OR } \\
\text { "Advance care planning" OR "Advance Directives" OR "Place of death" OR SU.EXACT("Palliative Care") } \\
\text { OR SU.EXACT("Hospice") OR SU.EXACT.EXPLODE("Life Sustaining Treatment") OR SU.EXACT("Advance } \\
\text { Directives") OR SU.EXACT.EXPLODE("Intellectual Development Disorder") OR SU.EXACT.EXPLODE("Drug } \\
\text { Abuse") OR SU.EXACT("Substance Use Disorder") OR "Mental Retardation" OR "Mental Deficiency" OR "Drug } \\
\text { Dependence" OR "Drug Addiction" OR "Substance Abuse" OR "Drug Abuse") }\end{array}$ & \\
\hline Limiters: & Spanish, English, scientific journals & \\
\hline Scopus & $\begin{array}{l}\text { (TITLE-ABS-KEY(“Ageing" OR “Older people" OR "Older adults” OR “Elderly" OR "Elders”)) OR } \\
\text { (INDEXTERMS(“Aged” OR "Aging”)) }\end{array}$ & 195 \\
\hline AND & $\begin{array}{l}\text { (TITLE-ABS-KEY(“Decision making" OR "Patient participation" OR "Patient Involvement” OR "Patient } \\
\text { Engagement”)) OR (INDEXTERMS(“Decision making" OR "Consensus" OR "Uncertainty" OR "Dissent and } \\
\text { Disputes" OR "Choice Behavior" OR "Negotiating" OR "Patient participation")) }\end{array}$ & \\
\hline AND & TITLE-ABS-KEY(“Relocation" OR “Placement" OR “Location of care” OR "Relocating" OR "Transition") & \\
\hline AND & $\begin{array}{l}\text { (TITLE-ABS-KEY(“Aging in Place” OR "Institutionalization" OR "Independent living")) OR } \\
\text { (INDEXTERMS(“Institutionalization" OR "Independent living" OR "Housing for the elderly" OR "Residential } \\
\text { facilities" OR "Assisted Living Facilities" OR "Homes for the Aged" OR "Nursing Homes")) }\end{array}$ & \\
\hline
\end{tabular}




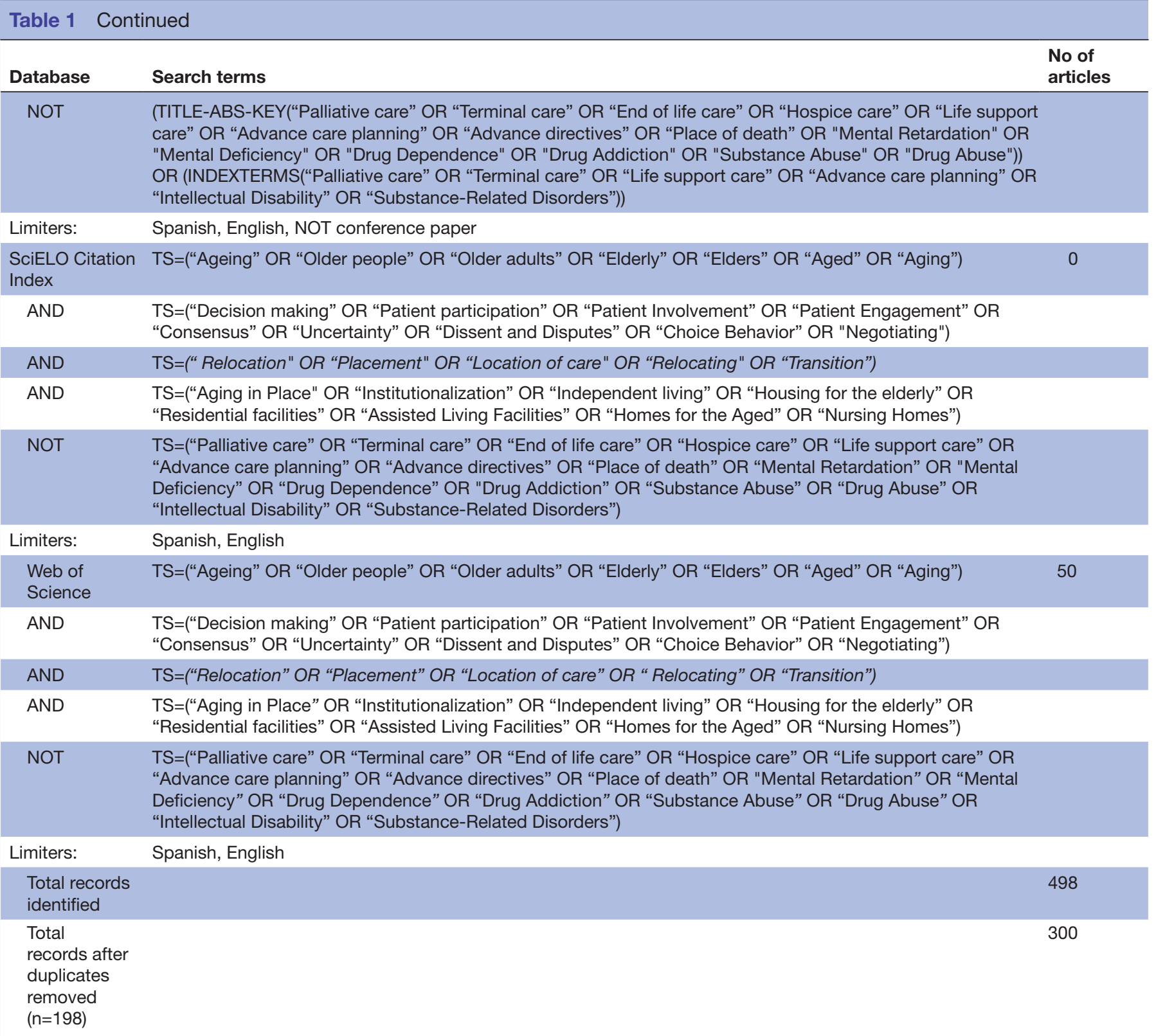

*PsycINFO: The search strategy provides 75 results, but only 74 are shown on the list. When the limit 'Scientific journals' is included, it provides 58 results, but only 57 are shown. When 'English' is introduced, it provides 55 results, but only 54 are shown. Therefore, the total amount of papers found on this database is 54 , since they are the ones we have access to, shown to us by the database.

specified, and the election of articles will not be filtered on the basis of that, but it will focus on the experiences of the participants throughout the process, thus being essential to understand the motives and/or reasons behind the decisions. In this way, it will be easier to understand in a more complete manner how this decision on the location of care of the elderly is made.

\section{Relocation process}

Generally speaking, the authors of this systematic review understand that the final decision after this decision-making process on the location of care may be simplified into two options: staying in the usual location of care or moving to a different place. In this review, both decision-making processes will be taken into account.
More precisely, as regards the decision of relocating, it is interesting to highlight a couple of aspects:

First, the literature describes different types of relocation, as the following examples mentioned by Smith and Crome $^{14}$ : home to institution, intrainstitutional and interinstitutional or institution to home, and these may be voluntary or involuntary, patients may be healthy or ill and the relocation may be well-planned or ill-planned. Yawney and Slover ${ }^{17}$ propose four types: from one community setting to another, from the community to an institution, from one institution to another and from an institution to the community.

Throughout this review, an institutional setting will be understood as those centres or institutions where the 
elderly are admitted to in order to receive care or supervision from healthcare professionals (eg, a nursing home for the elderly). On the other hand, the community setting, seen as the opposite of the institutional setting, includes homes, different types of housing or locations of care that are within the community context which promote or support the elderly's independent living (either if they have some help or supervision or not).

According to this, in this review we will deal with all relocations starting from the community setting, regardless of the chosen location of care, as long as they are permanent.

Second, different authors have described different phases in the relocation process, or, more precisely, in the institutionalisation process, ${ }^{18} 19$ the decision-making thus being an event taking place before the institutionalisation itself. This is why this review will only analyse and extract information linked to the phases of the process considering the moment where the decision of relocating is taken. Therefore, the phase where the participants are at, or whether the study deals with the complete institutionalisation process or only some phases, is irrelevant when it comes to including the studies in this review, since, in all these cases, only the information about the decision-making process will be taken into account.

\section{Location of care}

Lastly, as the different locations of care have been poorly and inconsistently described in the literature, ${ }^{20}$ it is important to point out that, throughout this review, the 'decision on the location of care' will be understood as any one that involves deciding on a permanent or longterm location of care for the elderly, regardless of the environment/place where they are relocated, either a home, community or institutional setting.

Therefore, all the decisions focusing on temporary locations of care, such us stays in hospitals, rehabilitation facilities or other healthcare centres, with the aim of tackling an acute or temporary healthcare problem, will not be taken into account, since hospitalisations due to severe illnesses/problems are described as inevitable, ${ }^{21}$ hospital care thus seen as necessary when patients are seriously ill. ${ }^{21} 22$

Therefore, the decisions on relocating to try to solve specific health problems (for instance, psychiatric inpatient care), decisions on where to take care of people with substance abuse problems or the decision of relocating people with intellectual disabilities will not be taken into account in this review, since the authors consider that the mentioned health problems are specific and extensive enough to constitute their own research questions.

On the other hand, this review will not include the studies linked to deciding the place where one wants to die either, since, as stated by Agar $e t a l^{23}$ the place of care must not be understood as the same thing as a place of death. Due to this, the studies dealing with decisions linked to the end of life, the care for the terminally ill, choosing the place where one wants to die, palliative care, advance care planning or advance directives will not be taken into account.

The eligibility criteria that will be used in the development of this systematic review are detailed below.

The studies eligible to be included in this review would be: (1) those dealing with decisions on the location of care of the elderly, adults who are 65 or older (when studies refer to decisions about a wide age range, the studies will be included if the average age is 65 or older, or if these studies analyse subgroups of people whose average age is 65 or older); (2) these studies must deal with the decision-making process on the location of care already experienced by the participants; (3) original studies; (4) qualitative or mixed-method studies; (5) written in English or Spanish (languages spoken by team members).

The studies will be excluded if they are: (1) studies where relocation has started in an institutional environment; (2) studies about deciding about the end of life, terminal patient care, palliative care, advance care planning, advance directives and/or the place to die; (3) studies about decisions on the location of care connected to substance abuse or intellectual disabilities; (4) studies about decisions on temporary locations of care, acute care and/or specific health problems, such as psychiatric inpatient care; (5) doctoral theses or conference proceedings (conference abstracts); (6) studies whose complete text is not accessible.

Qualitative research studies will not be limited by methodology (phenomenology, grounded theory, action research, ethnography, etc), while in the mixed-method studies only the qualitative components of the research will be included and analysed. However, those mixedmethod studies where it is not possible to tell if the results were obtained with quantitative or qualitative methods will be excluded.

\section{Data collection process}

The search results have been exported to the Zotero bibliography manager, in order to store, manage and organise the obtained bibliographical references. In addition, a register of the obtained results in the searches in each database has been kept.

The obtained citations have also been reviewed, deleting those which were repeated in the different databases.

Later, all these citations will be reviewed by title and abstract independently by two reviewers. These reviewers will meet to discuss the results and, in the case of disagreement, a third reviewer will mediate. All the doubtful citations will be included so that their complete text is read.

Finally, the complete text of all the included citations will be obtained, in order to assess if they must be included in the review. All the texts will be read in full and will be assessed by two reviewers independently, in order to decide if they must be included. These reviewers will meet to discuss the results and, in the case of disagreement, a third reviewer will mediate. If, after reading a complete paper or text, the information is not enough, 
or clear enough, the paper will finally be excluded from the review on the basis of the lack of information.

The above-mentioned steps will be reported using a flow chart.

\section{Extraction of data}

In order to identify the information on the results of the studies, we will follow a previous study on methods to thematically synthesise qualitative research in systematic reviews, and we will consider all text marked as 'results' or 'findings' in the papers to be the results of the studies, ${ }^{24}$ also adding all the text included under the title 'conclusions'. Both the participants' quotes and the authors' interpretations will be taken into account, since the extraction of this information (through both channels) helps to guarantee that the results obtained in the review are fully based on the real experiences of the participants in the studies, as proposed by Butler et al. ${ }^{11}$

To perform this task, a tool for data extraction specifically created for this review, based on the needs of our study, will be used, as proposed by Butler et al. ${ }^{11}$

This tool will be piloted with a small number of papers (from two to four) in order to check its usefulness, and it will be modified if required. The information to be extracted from each paper will be: title; year of publication; country; language; authors; objective of the study (main objective and, if applicable, secondary objectives); design: methodological basis; sample: strategy, size, inclusion and exclusion criteria, characteristics of the participants; techniques/methods for information collection; data analysis methods/techniques; ethical considerations; results: the participants' quotes and the authors' interpretations; final conclusion; strengths and limitations and comments by the reviewers.

All the obtained information will be classified into tables.

The extraction of information will be carried out by two reviewers independently. These reviewers will meet to discuss the results and, in the case of disagreement, a third reviewer will mediate.

\section{Quality appraisal}

The quality of the included studies will be assessed using Critical Appraisal Skills Programme Español (CASPe):Plantilla para ayudarte a entender un estudio cualitativo. ${ }^{25}$ This tool includes 10 questions designed to help to assess qualitative research studies, answering 'Yes', 'No' or 'Can't tell' to each question: the first two questions deal with the objectives of the research and the advisability of the qualitative methodology; these two questions are screening questions-only if the answer to both questions is 'Yes' it is worth proceeding with the remaining questions. ${ }^{25}$ The remaining questions deal with: the research method, the strategy for selecting participants, data gathering techniques, relationship between researcher and object of study, ethical issues, data analysis, exposure and applicability of the research results. ${ }^{25}$
This tool, in its English version, has already been used in different review papers. ${ }^{26-28}$ The tool will be tested with a sample of the studies to confirm that both reviewers are using it properly, as well as that the tool is clear and useful.

As regards the use of the studies' quality as an exclusion criteria, some authors in the bibliography choose to exclude papers from their studies according to their quality, ${ }^{26}{ }^{29}$ while others include all the papers. ${ }^{24}{ }^{27}$ Since the objective of our review is to provide an overview of how the location of care of the elderly is decided on, we will not exclude papers on the basis of their quality. ${ }^{11}$

However, the quality appraisal performed on each study included will be reflected, organising this information into a table. In addition, as the viability and importance of attempting some kind of sensitivity analysis will be a fundamental focus in future studies ${ }^{30}$ this systematic review will study the relative contributions of the different studies to the results of this review according to their quality, a process already performed by other authors before. $^{24}$

The critical quality appraisal will be carried out by two reviewers independently. These reviewers will meet to discuss the results and, in the case of disagreement, a third reviewer will mediate.

\section{Data synthesis}

The review we propose will be carried out with the aim of increasing the scientific production in the field of qualitative research and, more precisely, in the field of systematic reviews, since the inclusion of qualitative research in systematic reviews is still a big challenge. ${ }^{30}$ At the same time, the literature states the methods to synthetise and review evidence in order to tackle questions different from efficacy issues are much less developed. ${ }^{31}$

Dixon-Woods $e t a l^{2}$ state in their review about possible methods for qualitative and quantitative synthesis of evidence that the choice of synthesis type (either interpretive or integrative) is probably linked to the research question of the review. In addition, this review also points out different methods to synthesise the qualitative and quantitative evidence that might be used (narrative summary, grounded theory, meta-ethnography, meta-synthesis, meta-study, realist synthesis and Miles and Huberman's data analysis techniques, content analysis, case survey, qualitative comparative analysis and Bayesian meta-analysis). ${ }^{32}$

According to Butler et $a l,{ }^{11}$ the chosen synthesis method will depend on the review's type and goal. That same article also points out that, regardless of the chosen method, it is important to clearly report each of the steps, and how they are going to be taken, as this provides reproducibility, transparency and trust in the review's results. ${ }^{11}$

This is why, in order to synthesise the qualitative evidence included in this systematic review, a widely used and known method in the field of qualitative research will be used-the Constant Comparative Method ${ }^{33}$ from Glaser and Strauss' Grounded Theory. ${ }^{34}$ This method is being used today to synthesise qualitative evidence, ${ }^{35}$ and 
many researchers use it outside of Grounded Theory ${ }^{32}$ which is the approach chosen for this review.

The synthesis of data will be performed by two authors independently, and these results will be reviewed and discussed by all the authors in order to make sure that they suit the original information.

The software to be used to analyse all the information is Weft QDA.

\section{Patient and public involvement}

Patients and/or public are not involved in this study.

\section{Ethics and dissemination}

Thanks to this systematic review of the literature, we will achieve a deep understanding of how the decision on the location of care of the elderly is taken.

In addition, dealing with qualitative methodology studies will allow us to widely appraise the experiences that the main actors go through in this decision-making process that is so important in the elderly's lives which will help us to understand not only the reasons and emotions underlying this decision, but also to create new knowledge on the topic, useful both for researchers involved in this research field and all the people involved in this decision-making process today.

This work will also help to improve the field of study of systematic reviews of qualitative studies, since, in this research's final report, information will be provided on each of the steps taken to develop this systematic review which will help future researchers who wish to continue working on the review of qualitative studies.

Also, as this is a review unrestricted in time, it will allow us to analyse how this decision has been posed in time, in order to see if it has changed in the way of proceeding and in the emotions provoked by this choice in different time periods.

Our study can also have some limitations. The results obtained from this review will be limited by the inherent nature of qualitative research, apart from the limitations of the individual studies included. On the other hand, this protocol also has some limitations linked to the search strategy. There is no precise terminology to refer to the main key terms of the review; in addition, the used search terms had to be modified in order to adapt the strategy to each of the consulted databases, so maybe not all terms that might be linked to the topic of interest are found. Also, since it uses language limits, this review will not deal with research carried out in languages different from English or Spanish.

However, we have intended to assure the quality of this review protocol by leaning on the work by Butler $e t a l,{ }^{11}$ the PRISMA-P statement ${ }^{13}$ and the ENTREQ statement, ${ }^{12}$ the latter being the one to be used as a guide to develop the complete final systematic review. In addition, another tool will be used to check and report about the quality of the included studies, thus providing transparency and reliability to the review process. Also, it bears pointing out that, if any change were to be made in the process or the performed procedures, these would be clearly and precisely reported, providing due explanations and reasons.

The plans to disseminate the results of this systematic review include publishing a research paper in a high-impact journal in our study area. Also, if possible, the results of this research will be presented in scientific conferences. In addition, the obtained results will be suitable for informing, guiding, complementing and discussing the doctoral thesis of one of the authors of the review which is under way.

Acknowledgements The authors would like to thank reviewers for their valuable and useful advices and comments.

Contributors G-S-G conceived the study and the study design. G-S-G developed and executed the search strategy. G-S-G, R-S-d-R and M-R-R helped draft the protocol, edited the draft protocol, read and approved the final manuscript.

Funding The authors have not declared a specific grant for this research from any funding agency in the public, commercial or not-for-profit sectors.

Competing interests None declared.

Patient consent Not required.

Ethics approval This protocol did not require ethical approval, since it is a protocol for a systematic review.

Provenance and peer review Not commissioned; externally peer reviewed.

Open access This is an open access article distributed in accordance with the Creative Commons Attribution Non Commercial (CC BY-NC 4.0) license, which permits others to distribute, remix, adapt, build upon this work non-commercially, and license their derivative works on different terms, provided the original work is properly cited, appropriate credit is given, any changes made indicated, and the use is non-commercial. See: http://creativecommons.org/licenses/by-nc/4.0/.

\section{REFERENCES}

1. Organización Mundial de la Salud. Informe mundial sobre el envejecimiento y la salud. Ginebra: OMS, 2015.

2. Kogan AC, Wilber K, Mosqueda L. person-centered care for older adults with chronic conditions and functional impairment: a systematic literature review. J Am Geriatr Soc 2016;64:e1-e7.

3. Segunda Asamblea Mundial sobre el Envejecimiento. Declaración Política y Plan de Acción Internacional de Madrid sobre el Envejecimiento. Nueva York: Naciones Unidas, 2003. http://www.un. org/esa/socdev/documents/ageing/MIPAA/political-declaration-sp. pdf. (accessed 18 Nov 2017).

4. Carstensen LL, Hartel CR. When I'm 64. Washington, D.C: National Academies Press, 2006.

5. Sharp T, Moran E, Kuhn I, et al. Do the elderly have a voice? Advance care planning discussions with frail and older individuals: a systematic literature review and narrative synthesis. Br J Gen Pract 2013;63:e657-e668.

6. Wetzels R, Geest TA, Wensing M, et al. GPs' views on involvement of older patients: an European qualitative study. Patient Educ Couns 2004;53:183-8.

7. Bastiaens H, Van Royen P, Pavlic DR, et al. Older people's preferences for involvement in their own care: a qualitative study in primary health care in 11 European countries. Patient Educ Couns 2007;68:33-42.

8. Löckenhoff CE, Carstensen LL. Socioemotional selectivity theory, aging, and health: the increasingly delicate balance between regulating emotions and making tough choices. J Pers 2004;72:1395-424.

9. Stephens C, Breheny M, Mansvelt J. Healthy ageing from the perspective of older people: a capability approach to resilience. Psychol Health 2015;30:715-31.

10. The Joanna Briggs Institute. Joanna Briggs Institute Reviewers' Manual: 2014 edition. Australia: The Joanna Briggs Institute, 2014.

11. Butler A, Hall H, Copnell B. A Guide to Writing a Qualitative Systematic Review Protocol to Enhance Evidence-Based Practice in Nursing and Health Care. Worldviews Evid Based Nurs 2016;13:241-9. 
12. Tong A, Flemming K, Mclnnes $\mathrm{E}$, et al. Enhancing transparency in reporting the synthesis of qualitative research: ENTREQ. BMC Med Res Methodol 2012;12:181.

13. Shamseer L, Moher D, Clarke M, et al. Preferred reporting items for systematic review and meta-analysis protocols (PRISMA-P) 2015: elaboration and explanation. BMJ 2015;349:97647.

14. Smith AE, Crome P. Relocation mosaic - a review of 40 years of resettlement literature. Rev Clin Gerontol 2000;10:81-95.

15. Dickinson D. Can elderly residents with memory problems be prepared for relocation? J Clin Nurs 1996;5:99-104.

16. Matteson P, Hawkins JW. Concept analysis of decision making. Nurs Forum 1990;25:4-10.

17. Yawney BA, Slover DL. Relocation of the elderly. Soc Work 1973;18:86-95.

18. Kasl SV. Physical and mental health effects of involuntary relocation and institutionalization on the elderly--a review. Am J Public Health 1972;62:377-84.

19. Tobin SS, Lieberman MA. Last home for the aged: Critical implications of institutionalization. Ann Arbor: University Microfilms International, 1991.

20. Boland L, Légaré F, Perez MM, et al. Impact of home care versus alternative locations of care on elder health outcomes: an overview of systematic reviews. BMC Geriatr 2017;17:20.

21. Hallgren J, Ernsth Bravell M, Dahl Aslan AK, et al. In Hospital We Trust: Experiences of older peoples' decision to seek hospital care. Geriatr Nurs 2015;36:306-11.

22. Fried TR, van Doorn C, O'Leary JR, et al. Older person's preferences for home vs hospital care in the treatment of acute illness. Arch Intern Med 2000;160:1501-6.

23. Agar M, Currow DC, Shelby-James TM, et al. Preference for place of care and place of death in palliative care: are these different questions? Palliat Med 2008;22:787-95.

24. Thomas J, Harden A. Methods for the thematic synthesis of qualitative research in systematic reviews. BMC Med Res Methodol 2008;8:45.

25. Cano Arana A, González Gil T, Cabello López JB, et al. Plantilla para ayudarte a entender un estudio cualitativo. In: CASPe. Guías
CASPe de Lectura Crítica de la Literatura Médica. Alicante: CASPe: Cuaderno III, 2010:3-8.

26. Lang H, France E, Williams B, et al. The psychological experience of living with head and neck cancer: a systematic review and metasynthesis. Psychooncology 2013;22:2648-63.

27. Davies N, Maio L, Rait G, et al. Quality end-of-life care for dementia: What have family carers told us so far? A narrative synthesis. Palliat Med 2014;28:919-30.

28. Zheng R, Lee SF, Bloomer MJ. How new graduate nurses experience patient death: A systematic review and qualitative meta-synthesis. Int J Nurs Stud 2016;53:320-30.

29. Abad-Corpa E, Gonzalez-Gil T, Martínez-Hernández A, et al. Caring to achieve the maximum independence possible: a synthesis of qualitative evidence on older adults' adaptation to dependency. $J$ Clin Nurs 2012;21:3153-69.

30. Dixon-Woods M, Bonas S, Booth A, et al. How can systematic reviews incorporate qualitative research? A critical perspective. Qualitative Research 2006;6:27-44.

31. Popay J, Roberts H. Introduction: methodological issues in the synthesis of diverse sources of evidence. In: Popay J, ed. Moving beyond effectiveness in evidence synthesis: Methodological issues in the synthesis of diverse sources of evidence. London: National Institute for Health and Clinical Excellence, 2006:1-4.

32. Dixon-Woods M, Agarwal S, Jones D, et al. Synthesising qualitative and quantitative evidence: a review of possible methods. J Health Serv Res Policy 2005;10:45-53.

33. Glaser BG. The Constant Comparative Method of Qualitative Analysis. Soc Probl 1965;12:436-45.

34. Glaser BG, Strauss AL. The Discovery of Grounded Theory: Strategies for Qualitative Research. New York: Aldine Publishing Company, 1967.

35. de la Cuesta-Benjumea C, Henriques MA, Abad-Corpa E, et al. "Falls prevention among older people and care providers: protocol for an integrative review". J Adv Nurs 2017;73:1722-34. 